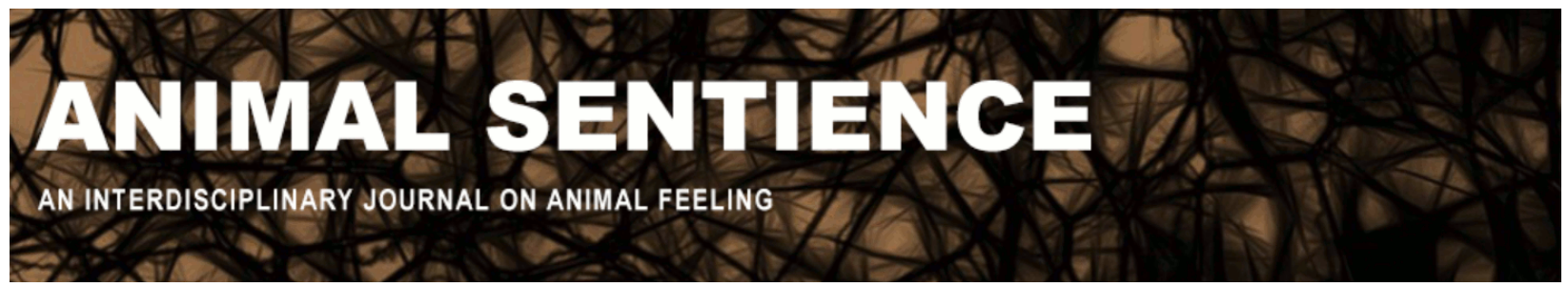

Reber, Arthur S. (2016) Resolving the hard problem and calling for a small miracle. Animal Sentience 11(9)

DOI: $10.51291 / 2377-7478.1175$

Date of submission: 2016-11-07

Date of acceptance: 2016-11-18 (c) 


\title{
Resolving the hard problem and calling for a small miracle
}

\author{
Response to Commentary on Reber on Origins of Mind
}

\author{
Arthur S. Reber \\ Department of Psychology, University of British Columbia
}

\begin{abstract}
With the exception of the commentary by Key, the commentaries on Reber have a common feature: the commenters feel, with varying levels of enthusiasm, that there is at least some virtue in the core assumption of the Cellular Basis of Consciousness (CBC) theory that consciousness (or subjectivity or sentience) accompanies the earliest forms of life. The model has two important entailments: (a) it resolves the (in)famous Hard Problem by redirecting the search for the biochemical foundations of sentience away from human consciousness; and (b) it reduces the need for an emergentist miracle to a far simpler scale than is currently assumed. The CBC is grounded in classical principles of evolutionary biology, which it shares with allied areas of research on emotion, learning, memory and perception.
\end{abstract}

Arthur S. Reber is Broeklundian Professor of Psychology, Emeritus, Brooklyn College and Graduate Center of the City University of New York, and currently Visiting Professor, Psychology, University of British Columbia. His research is on implicit learning, the process through which knowledge about the world about us is picked up largely independently of awareness of both the process and products of that learning. http://academic.brooklyn.cuny.edu/userhome/psych/areber/

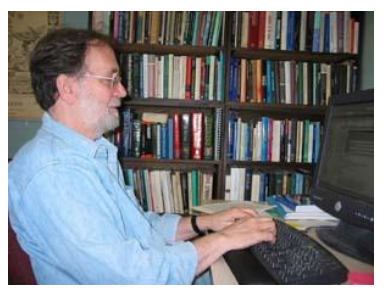

I am pleased to find some common ground with Brakel Woodruff, Velmans, Ristau,

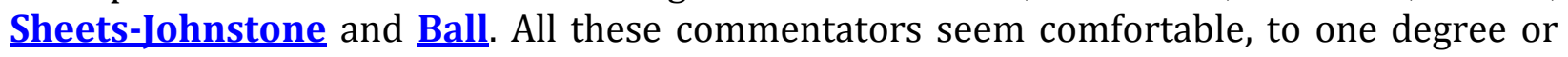
another, with what was once thought to be a wildly radical idea. Key takes issue. I'll briefly address each.

Brakel feels that I still need to worry about two additional "gaps," an epistemological gap (the relation between the cellular level and subjective mental outcomes) and a causal gap (how the cellular processes cause the mental). I have no problem with this way of putting things - provided that Brakel is comfortable with a simple edit. I deleted "brain" from what she wrote. If we leave it in we're going to be back wrestling with Chalmers's hard problem. The point of the CBC isn't to duck or "finesse" these issues but to note that, by acknowledging the evolutionary starting point and focusing on the bio-evolutionary mechanisms of speciation, these "gaps" are more likely to be bridged.

There are hints of progress on the evolutionary mechanisms operating here (see the overview in Godfrey-Smith, 2016). Discovering the protein-folding mechanisms that allow for the experience of "good to consume" or "need to recoil" will be a lot easier than trying to understand how the myriad, interlocking cortical and subcortical pathways and centers in the human brain produce the sense of "me-ness." 
Woodruff begins by questioning the central proposition of the CBC model. Yet, oddly, as I read his comment, it appears that he might actually think it's correct — or, if not, then at least closer to being correct than other extant theories. The problem is that the counter-evidence he presents doesn't seem to be contrary to the CBC. First, the example of Hox genes seems irrelevant since they don't appear evolutionarily until more complex species like Drosophia and others with segmented bodies. The CBC begins with single-cell organisms.

Woodruff's other example - bacteria with flagella - is precisely what I'm focused on. The genetic mechanisms that control the actions of the flagella seem to be very much the result of a primitive sentience. The emergence of the genes that operate in these contexts was almost certainly contingent upon the pre-flagellum bacteria being sensitive to the toxic or beneficial qualities of the objects they came into contact with. The flagellum was the structure that evolved to control adaptive locomotion. Pure continuity here is not a requirement (a point also made by Ristau - see below). We only need to see a common mechanism operating.

Woodruff's claim that "genetic analysis also fails to show species continuity underlying bio-sensitivity in bacteria" seems a bit premature to me since we don't yet know what the genetic foundations for bio-sensitivity are. Bio-philosopher Godfrey-Smith (2016) argues that the place to look for the origins of subjectivity is the prokaryotes. He traces the biological mechanisms of single-celled organisms with a Nagel-like question, "is there anything it is like to be a bacterium?" Godfrey-Smith's program is ambitious as he is also concerned with issues such as individuality or at what point multi-cellular organisms can be considered distinct organisms rather than collections of independent cells (see Godfrey-Smith, forthcoming).

Woodruff also worries about parsimony. I do not think the CBC model makes us "set aside" Occam's razor. If anything, the model reflects a passionate embrace of the Venerable William for it reduces the search for an essential, material aitia (pacé Aristotle) to a single biological structure. And, as Ristau noted, Occam's razor is really a pragmatic principle.

Velmans, as always, presents incisive and creative perspectives. His distinction between "continuity" and "discontinuity" theories is intriguing. I suppose there's no shame that whereas I had thought I was presenting a continuity model, I discover that I am just another "discontinuitist" who needs a bigger miracle than I thought.

But Velmans wants more. He hints at the possibility of including plants within the CBC umbrella (as does Ristau). Again, I could live with that. Should biological evidence emerge that species with rigid cell walls do have subjective, sentient states, I'd welcome it. I'd wager against it but would certainly be comfortable with the discovery that there is indeed "something it is like" to be a Mimosa or a "snap trap" carnivorous plant like Dionaea muscipula. It's an empirical question, not a theoretical one.

But Velmans would still not consider this revised CBC model a continuity theory for it would still call for determining the specific biological conditions that allow for sentience. He hints at (and develops more completely in an unpublished paper that can be linked here) the need for a fully formed continuity theory grounded on the panpsychic notion that sentience is an inherent feature of the universe. 
This position has obvious problems. For one, if sentience is everywhere it might as well be nowhere for it will be impossible to ascertain either existence or nonexistence. Moreover, while the $\mathrm{CBC}$ requires a modest miracle, Velmans will need an enormous one. He will also need to explain why this cosmic sentience, so far as we can tell, only shows up in entities that are alive.

Ristau's first objection strikes me as not an objection at all. She is uncomfortable with the analogy to physics, noting that we don't really know what it means to say that gravity is an inherent feature of mass. Precisely. We don't. But that doesn't corrupt the analogy. We also don't know what features of primitive organic forms will prove to be the inherent features that produce subjectivity. That, too, is a scientific question.

Ristau, like Velmans, is open to the possibility that some plant species may have rudimentary forms of sentience, for example, aquatic species that develop sensitivity to nutrients in currents. Again, I am comfortable extending the CBC to include these species. The critical evidence will of course come when we unravel the genetic basis for primitive sentience and determine whether both groups of species share it.

Sheets-Johnstone and I appear to agree on both the core axiom of the CBC and the notion that locomotion is a critical feature. She chides me for not going back to Darwin for common ideas and similar language. I appreciate her comment and take it as a compliment.

Key is the only commentator so far who has a fundamental disagreement with my thesis. The heart of his argument is found here: "... it is rather a long bow to draw to suggest that organisms without a nervous system possess subjective awareness...."

In fact, it is not. Key's position is consonant with the one he took elsewhere with regard to the experience of pain (Key, 2016a,b,c,d). Many of the comments on his thesis point out the compelling, cross-species similarity in the manner and mode of expression of emotions, noting that (as Sheets-Iohnstone would counsel) they are similar to arguments presented effectively by Darwin (1872). As I noted in the target article, I do not shrink from what I view as reasoned anthropomorphism. I find it unfathomable that the fish flopping around in the bottom of a boat desperately seeking oxygen is not in pain and that its suffering is not on a continuum with mine.

The really "long bow to draw" is the one into which Key is trying to fit an arrow. It requires that at some moment in evolutionary history a capacity for subjective representation blinked into ontogenetic reality whereas one cosmic moment before no species possessed it. A position that is far more coherent biologically is to view the pain felt by organisms with complex nervous systems as lying along an evolutionary lineage from earlier species that experienced subjective states with an "unpleasant" quale. There is, in fact, "something it is like" to be a spider (or a sea anemone or a prokaryote) in pain (cf. Klein \& Barron, 2016).

Oddly, Key seems not to grasp a fundamental principle: Functions can evolve - and often have - more than once, taking different evolutionary paths. Insects, birds and mammals all have evolved flight independently. That human pain has identifiable neural pathways and centers does not mean that these structures are the only ones that mediate true pain. That human consciousness has its particular characteristics and associated neurological foundations does not mean that there are not other ways of instantiating a "what it's like to be" subjective state. 
Although I didn't touch on it in the target article, the CBC model is part of a larger theoretical framework of the ontogeny and phylogeny of cognitive functions primarily focused on implicit learning (Reber, 1989, 1992a,b, 1993). The theory is founded on four classic heuristics of evolutionary biology:

1. Success. Once adaptive forms emerge, they become the foundation for later forms.

2. Conservation. Evolution is conservative. Adaptive forms and functions tend to become fixed and serve as platforms for emerging forms.

3. Stability. Early-appearing, adaptive forms and structures tend toward stability, showing fewer successful variations than later-appearing forms and less individual-to-individual variation.

4. Commonality. Evolutionarily older forms and functions will be displayed across species.

The arguments in the target article are consonant with these principles. The sentience that is hypothesized to be an inherent feature of single-cell organisms is a function that emerged early in evolutionary time, was adaptive for a mobile species, was conserved in later-evolving species, is stable, maintains its capacity to inform about the valences of critical features of the environment and shows commonality across species.

As noted in the target article, evolution has a pyramidal character. The older forms and functions lie at a broad base from which successive forms and functions evolve. Mutations and variations tend to occur in the upper layers, not in the core, foundational form. Once an adaptive form evolved, the fundamental features remained stable. Additional features were incorporated, complexity was added, novel functions emerged but an essential commonality across species was maintained. See Hodos (2009) on the notochord and how it formed the foundation for the evolution of the many variations on spinal cords and vertebrae while holding fast to the original structure. The heart of my argument is that consciousness, subjectivity, sentience is like that.

Key also chides me for failing to "appreciate that novel and significant form and function can emerge any time during evolution through genetic modifications." He notes that "The power of any good hypothesis is revealed by its ability to (i) make new predictions that are experimentally testable and capable of advancing knowledge, and (ii) explain existing knowledge and future observations." And that the CBC fails on both.

I appreciate that Key is almost certainly unaware of my earlier theoretical and empirical work. Not only did that theory of the cognitive unconscious predict the emergence of "novel and significant form and function," it made important "new predictions" and "explained existing knowledge" about how unconscious cognitive functions are manifested within humans and across species.

The work has had a lasting impact. Lancaster University hosts an annual conference on implicit learning. There is a Handbook of Implicit Learning (Stadler \& Frensch, 1998), and there are literally scores of books and thousands of papers in diverse areas following up on the original research. One can only hope that the CBC will generate similar interest. 
The Hard Problem: The way I tried to dispatch Chalmers's (in)famous conundrum is called into question by Ball, Brakel and Woodruff. When I stated that the hard problem was the victim of a category error, I meant that Chalmers (1995) erred when he treated human consciousness as ontologically distinct and epistemically separate from the forms of consciousness expressed in other, less cellularly complex species. His formulation stimulated fascinating and creative efforts to unravel how brains make minds (which I briefly reviewed) - none of which have come anywhere near solving the problem.

There is nothing in the $\mathrm{CBC}$ that minimizes or diminishes the value of this research. Even when we know how ur-consciousness emerges we will still need to understand the bio-mechanisms that produce the manifold forms of sentience we see developing along the evolutionary continuum. Similar research strategies are in place for other domains of study such as memory, learning, perception and emotion.

I guess, my move doesn't "solve" the hard problem but it does "resolve" it. Amusingly, it fits with Chalmers's own view. He thinks that among the "easy" problems is ascertaining the mechanisms that give rise to the ability to react to environmental stimuli - he just didn't want to call this "consciousness."

Ball is right that the CBC model contains an invitation to adopt a particular research strategy, one driven by reductionist principles that counsel focusing on basic bio-genetic issues. He also argues that I haven't solved the "hard problem" - which is fine because I wasn't trying to. The goal is to "re-solve" it by focusing on foundational issues that are far less refractory than the ones Chalmers put before us. But Ball phrases his critique in an odd manner. He maintains that the hard problem contains within it the need to answer questions like, "Why does this (or that) state feel the way it does?" "Why do bacteria feel the way they do, rather than some other way or no way at all?" - and that the CBC has no answer.

But there was no attempt to answer these questions. They are seductive questions, philosophically engaging ones but, at their heart, they are empirical questions. My point is that we have a higher likelihood of finding answers by taking a reductionist, evolutionarybiological stance. Single-cell organisms feel. Feelings are subjective states. There is something it is like and feels like to be a bacterium. The first order of business is to work out the protein folding mechanism(s) that give rise to these kinds of subjective states.

\section{References}

Ball, D. (2016). No help on the hard problem. Animal Sentience 2016.149.

Brakel, L. A. W. (2016). Mind/body "hard problem" is not a category error. Animal Sentience 2016.131.

Chalmers, D. (1995). Facing up to the problem of consciousness. Journal of Consciousness Studies, 2, 200-219.

Darwin, C. (1872). The Expression of the Emotions in Man and Animals. London: John Murray.

Godfrey-Smith, P. (2016). Animal evolution and the origins of experience. In D. L. Smith (Ed.), How Biology Shapes Philosophy. NY: Cambridge University Press. 
Godfrey-Smith, P. (forthcoming). Individuality, subjectivity and minimal cognition. Biology and Philosophy.

Hodos, W. (2009). Evolution of the spine. In M. D. Binder, N. Hirokawa \& U. Windhorst (Eds.), Encyclopedia of Neuroscience (pp. 1421-1424). Berlin: Springer.

Key, B. (2016a). Why fish do not feel pain. Animal Sentience 2016.003.

Key, B. (2016b). Going beyond just-so stories. Animal Sentience 2016.022.

Key, B.(2016c). Falsifying the null hypothesis. Animal Sentience 2016.39.

Key, B. (2016d). Burden of proof lies with proposer of celestial teapot hypothesis. Animal Sentience 2016.079.

Key, B. (2016e). "Cellular basis of consciousness": Not just radical but wrong. Animal Sentience 2016.137.

Klein, C. \& Barron, A.B. (2016). Insects have the capacity for subjective experience. Animal Sentience 2016.100.

Land, M. F. \& Fernald, R. D. (1992). The evolution of eyes. Annual Review of Neuroscience, 15, 1-29.

Reber, A. S. (1989). Implicit learning and tacit knowledge. Journal of Experimental Psychology: General, 118, 219-235.

Reber, A. S. (1992). The cognitive unconscious: An evolutionary perspective. Consciousness and Cognition, 1, 93-133.

Reber, A. S. (1993). Implicit learning and tacit knowledge: An essay on the cognitive unconscious. NY: Oxford University Press.

Reber, A. S. (2016). Caterpillars, consciousness and the origins of mind. Animal Sentience 2016.106.

Ristau, C. A. (2016). Beginnings: Physics, sentience and LUCA. Animal Sentience 2016.134.

Sheets-Johnstone, M. (2016). Darwin's empirical evidence. Animal Sentience 2016.140.

Stadler, M. A. \& Frensch, P. A. (Eds.) (1998). Handbook of Implicit Learning. Thousand Oaks: Sage.

Velmans, M. (2016). How could consciousness emerge from adaptive functioning? Animal Sentience 2016.138.

Woodruff, M. L. (2016). Bacteria and the cellular basis of consciousness. Animal Sentience 2016.126. 